\title{
Cognitive functioning in glioblastoma patients during radiotherapy and temozolomide treatment: initial findings
}

\author{
Karen Hilverda $\cdot$ Ingeborg Bosma $\cdot$ Jan J. Heimans $\cdot$ \\ Tjeerd J. Postma $\cdot$ W. Peter Vandertop - Ben J. Slotman • \\ Jan Buter · Jaap C. Reijneveld · Martin Klein
}

Received: 6 February 2009/Accepted: 10 August 2009/Published online: 30 August 2009

(C) The Author(s) 2009. This article is published with open access at Springerlink.com

\begin{abstract}
The aim of this study was to evaluate cognitive functioning in newly-diagnosed glioblastoma multiforme (GBM) patients during treatment with radiotherapy (RT) plus concomitant and adjuvant temozolomide (TMZ). Cognitive assessment took place following surgery, but prior to the start of RT (baseline), after 6 weeks of RT and concomitant TMZ (1st follow-up), and after three cycles of adjuvant TMZ (2nd follow-up). Standardized cognitive summary measures and delta scores for six cognitive domains were calculated at the individual level. Cognitive functioning of progression-free GBM patients was compared to that of matched healthy controls. Analyses were
\end{abstract}

K. Hilverda $(\bowtie) \cdot$ M. Klein

Department of Medical Psychology, VU University Medical Center, Van der Boechorststraat 7, 1081 BT Amsterdam,

The Netherlands

e-mail: k.hilverda@vumc.nl

I. Bosma - J. J. Heimans - T. J. Postma - J. C. Reijneveld Department of Neurology, VU University Medical Center,

De Boelelaan 1117, 1081 HV Amsterdam, The Netherlands

W. Peter Vandertop

Department of Neurosurgery, VU University Medical Center, De Boelelaan 1117, 1081 HV Amsterdam, The Netherlands

B. J. Slotman

Department of Radiation Oncology, VU University Medical Center, De Boelelaan 1117, 1081 HV Amsterdam,

The Netherlands

J. Buter

Department of Medical Oncology, VU University Medical

Center, De Boelelaan 1117, 1081 HV Amsterdam,

The Netherlands

J. C. Reijneveld

Department of Neurology, Academic Medical Center,

Meibergdreef 9, 1100 DD Amsterdam, The Netherlands performed on a group of 13 GBM patients that were progression-free during follow-up. The results showed that the majority of patients had deficits in multiple cognitive domains at baseline. Between baseline and 1st follow-up, four patients improved in one cognitive domain, four patients deteriorated in one domain, one patient improved in one domain and deteriorated in another, and four patients remained stable in all six domains. Between 1st and 2nd follow-up, the majority of patients (11) remained stable in all six cognitive domains, whereas one patient declined in one domain, and one patient showed a deterioration in two domains. Overall, between baseline and 2nd follow-up, three patients improved in one cognitive domain, two patients deteriorated in two domains, one patient improved in one domain and deteriorated in another, and seven patients remained stable in all six cognitive domains. In conclusion, preceding treatment, the majority of GBM patients show clear-cut deficits in cognitive functioning. In the course of the first 6 months of their disease, however, progression-free GBM patients undergoing radiotherapy plus concomitant and adjuvant temozolomide treatment do not deteriorate in cognitive functioning.

Keywords Cognitive functioning .

Glioblastoma multiforme $\cdot$ Radiotherapy $\cdot$ Temozolomide

\section{Introduction}

Glioblastoma multiforme (GBM) is the most aggressive primary brain tumor. As GBM patients cannot be cured, treatment procedures primarily focus on extending life expectancy and palliation of symptoms. Recently, a large international randomized trial has shown that the addition of concomitant and adjuvant temozolomide (TMZ) to 
radiotherapy (RT) results in a modest but significant 2.5month survival benefit with minimal additional toxicity. The 2-year survival rate was $26.5 \%$ with RT plus TMZ and $10.4 \%$ with RT alone [1].

However, the prognosis of GBM patients still remains poor, with all patients eventually dying due to tumor progression. Therefore, the quality of survival of these patients is of major importance. Cognitive deficits, potentially compromising health-related quality of life, are commonly observed in GBM patients in different stages of the disease [2]. It is known that cognitive deficits exist before therapy starts [3, 4], and remain or worsen in the course of the disease $[5,6]$. A recent study reported a marked decline in cognitive functioning during and following RT treatment in high-grade glioma patients who received RT only as upfront treatment. The cognitive decline was more pronounced in patients with tumor recurrence compared to progression-free patients, which could be attributed to the use of anti-epileptic drugs [7].

As treatment methods keep changing and becoming more aggressive, monitoring possible treatment sideeffects on cognitive functioning remains necessary. An important question is how cognitive deficits may develop during this intensified RT and TMZ treatment, compared to treatment with RT alone, since literature suggests that more central nervous system toxicity as expressed by radiological abnormalities, blood-brain barrier disruption, and cognitive deficits, may ensue due to intensified treatment [8].

While health-related quality of life in newly-diagnosed GBM patients has already been reported to remain stable during a regimen of RT and TMZ treatment [9], this study aims at determining whether this also holds for cognitive functioning. Therefore, the purpose of the present study is to prospectively examine cognitive functioning in newlydiagnosed, progression-free GBM patients, at different points in time during combined radio-chemotherapy.

\section{Methods}

\section{Patients}

Consecutive, newly-diagnosed GBM patients were recruited from the VU University Medical Center, Amsterdam, The Netherlands, between March 2005 and June 2007. The inclusion criteria were: (1) histologically confirmed GBM, (2) no former treatment with radiation or chemotherapy, (3) age $>18$ years, and (4) able to communicate in the Dutch language. After the study protocol was approved by the local ethics committee, informed consent procedures were carried out in the postsurgical period, before the start of radiotherapy.
The treatment regimen was described in detail by Stupp et al. [1]. In short, all patients underwent neurosurgery (biopsy or resection) and subsequent treatment with 6 weeks of RT plus concomitant TMZ and six cycles of adjuvant TMZ. Patients participated in three assessments of cognitive functioning and magnetic resonance imaging (MRI): (1) after surgery, but before RT and concomitant TMZ, (2) after 6 weeks of conventional RT and concomitant TMZ, but before adjuvant TMZ, and (3) after three cycles of adjuvant TMZ. Patients with tumor progression during treatment were excluded from statistical analyses, to avoid the possible effects of tumor recurrence on cognitive functioning.

Normative data of healthy controls were used as a reference point for interpreting GBM patients' neuropsychological test results. These healthy controls were individually matched with GBM patients with respect to age, sex and educational level.

\section{Outcome measures}

Cognitive functioning was assessed by a battery of standardized tests. Because of the heterogeneity of both origin and severity of cognitive impairments in GBM patients, the battery consisted of a wide variation of tests assessing multiple cognitive domains. Trained psychometricians administered a neuropsychological test battery consisting of the following tests: Letter digit substitution test [10], Stroop color-word test [10], Concept shifting test [11], Categoric word fluency test [10], Visual verbal learning test [10], and Working memory task [12]. To accomplish data reduction, cognitive summary measures were calculated to detect possible deficits in the cognitive domains of (1) information processing speed, (2) psychomotor function, (3) attention, (4) verbal memory, (5) working memory, and (6) executive functioning (Table 1). Construction of these domains was based on a principal component analysis performed on the performances of healthy controls. Standardized cognitive summary measures ( $z$-scores) and delta scores (as a measure of change over time) for all six cognitive domains were calculated at the individual level.

\section{Statistical analysis}

In line with standards used in neuropsychological practice, an individual $z$-score of 1.5 or more below that of healthy controls $(z \leq-1.5)$ was defined as a clinically significant deficit in cognitive functioning. Furthermore, a change of $1.5 z$-score or more $(z$ (delta) $\geq 1.5$ or $z$ (delta) $\leq-1.5$ ) was defined as a clinically significant improvement or deterioration. 
Table 1 Cognitive domains and tests used

\begin{tabular}{ll}
\hline Domain & Test \\
\hline Information processing speed & Letter digit substitution test (writing condition and reading condition) \\
Psychomotor function & Concept shifting test (condition 0); Letter digit substitution test ( $\Delta$ score: writing condition-reading condition) \\
Attention & Stroop color-word test (word condition, color condition, color-word condition, and interferention score) \\
Verbal memory & Visual verbal learning test (first trial, total of five trials, $\Delta$ score trial 5-trial 1, active delayed recall, \\
& and delayed recognition) \\
Working memory & Working memory task (condition \%, conditions with 1 letter, 2 letters, 3 letters, and 4 letters) \\
Executive functioning & Concept shifting test (condition a (numbers), b (letters), c (number-letter)); Categoric word fluency test
\end{tabular}

\section{Results}

\section{Patient characteristics}

Analyses were performed on a total of 13 GBM patients. Six additional patients were excluded because of clinical and/or radiological tumor progression. All included patients were progression-free up to at least three cycles of adjuvant TMZ treatment. Table 2 lists the sociodemographic and clinical characteristics of these patients.

\section{Changes in anti-epileptic drug use and dexamethasone use}

For some patients, changes in baseline AED and dexamethasone use (shown in Table 2) occurred during RT and TMZ treatment. Patient 5 was using AED at baseline, but continued having frequent seizures. Therefore, the dose of valproic acid was increased during RT and concomitant TMZ treatment and once again during the first cycle of adjuvant TMZ. For patient 9, the dose of phenobarbital was increased during RT and concomitant TMZ because of persistent seizures, the dose of valproic acid remained unchanged. Patient 10 did not use any AED at baseline, but experienced a seizure during the second adjuvant cycle of TMZ and therefore started using valproic acid. The dose of levetiracetam of patient 11 was escalated during RT and concomitant TMZ because of an increase in partial seizures.

As to the changes in dexamethasone use, patient 3 started using dexamethasone just before the first adjuvant cycle of TMZ, because of headaches, and continued taking a low dose of dexamethasone during all adjuvant cycles. Patient 9 also began using dexamethasone because of

Table 2 Clinical variables

\begin{tabular}{|c|c|c|c|c|c|c|c|c|c|c|c|c|}
\hline \multirow[t]{2}{*}{ Case } & \multirow[t]{2}{*}{ Age } & \multirow[t]{2}{*}{ Sex } & \multirow[t]{2}{*}{ Tumor location } & \multirow[t]{2}{*}{$\begin{array}{l}\text { Neurosurgical } \\
\text { intervention }\end{array}$} & \multirow[t]{2}{*}{$\begin{array}{l}\text { Epilepsy } \\
\text { burden }^{\mathrm{a}}\end{array}$} & \multicolumn{2}{|l|}{$\begin{array}{l}\text { Anti-epileptic } \\
\text { drugs }\end{array}$} & \multicolumn{2}{|c|}{ Dexamethasone } & \multicolumn{3}{|c|}{$\begin{array}{l}\text { Karnofsky } \\
\text { performance } \\
\text { scale }\end{array}$} \\
\hline & & & & & & B & FU & B & FU & B & FU1 & FU2 \\
\hline 1 & 36 & Male & Right parietotemporal & Resection & 5 & Valproic acid & Unchanged & No & No & 90 & 90 & 100 \\
\hline 2 & 67 & Male & Right temporal resection & Resection & 5 & Valproic acid & Unchanged & No & No & 90 & 100 & 100 \\
\hline 3 & 63 & Female & Left parietotemporal & Resection & 1 & None & Unchanged & No & Yes & 60 & 60 & 60 \\
\hline 4 & 50 & Female & Left frontotemporal & Resection & 1 & None & Unchanged & No & No & 90 & 90 & 100 \\
\hline 5 & 51 & Male & Right parietooccipital & Biopsy & 5 & Valproic acid & Dose $\uparrow$ & No & No & 100 & 90 & 90 \\
\hline 6 & 58 & Male & Right frontal & Biopsy & 1 & None & Unchanged & No & No & 90 & 90 & 100 \\
\hline 7 & 32 & Male & Left parietooccipital & Resection & 1 & None & Unchanged & Yes & Yes & 90 & 90 & N/A \\
\hline 8 & 61 & Male & Left temporal & Biopsy & 5 & Phenitoin & Unchanged & No & No & 90 & 90 & 90 \\
\hline 9 & 18 & Male & Right parietotemporal & Resection & 6 & $\begin{array}{l}\text { Valproic acid } \\
\text { Phenobarbital }\end{array}$ & Dose $\uparrow$ & No & Yes & 100 & 90 & 90 \\
\hline 10 & 63 & Male & Right frontoparietal & Biopsy & 1 & None & Valproic acid & Yes & No & 70 & 70 & 70 \\
\hline 11 & 55 & Male & Left parietal & Resection & 6 & Levetiracetam & Dose $\uparrow$ & No & Yes & 70 & 90 & 90 \\
\hline 12 & 63 & Male & Left frontal & Biopsy & 1 & None & Unchanged & No & No & 90 & 90 & 90 \\
\hline 13 & 72 & Male & Right frontal & Biopsy & 5 & Valproic acid & Unchanged & No & No & 90 & 90 & 80 \\
\hline
\end{tabular}

${ }^{a}$ Epilepsy burden: 1 = epilepsy free, 5 = epilepsy, $<6$ seizures in previous year and on anti-epileptic drugs (AED) mono- or polytherapy, $6=$ epilepsy, $>6$ seizures in previous year and on AED mono- or polytherapy

$B$ Baseline, after surgery, but before RT and TMZ treatment, $F U$ regular, ongoing medical examinations during RT and TMZ treatment (the exact moment of changes during follow-up are described in the text), FU1 follow-up 1 (after 6 weeks of conventional RT and concomitant TMZ, but before adjuvant TMZ), FU2 follow-up 2 (after three cycles of adjuvant TMZ), N/A data not available 
headaches, but started during RT and concomitant TMZ, and continued his use until the second adjuvant cycle of TMZ. Patient 11 started dexamethasone use during the first cycle of adjuvant TMZ because of edema and problems in word finding, and continued using dexamethasone during all adjuvant cycles of TMZ. The clinical complaints of these three patients diminished by using dexamethasone and none of the patients showed radiological signs of tumor progression.

\section{Cognitive performance}

Cognitive functioning was tested in all 13 patients. One patient (case 3 ) could not attend to all tests, due to visual deficits. $z$-scores and delta-scores of all patients are shown in Table 3.

\section{Cognitive performance at baseline}

Compared to matched healthy controls, 11 patients had deficits (previously defined as $z \leq-1.5)$ in one $(n=2)$ or multiple $(n=9)$ cognitive domains at baseline, while 2 patients performed comparable to the healthy population. Most deficits were found in information processing speed $(n=8)$, executive functioning $(n=8)$ and attention $(n=6)$. Fewer deficits were found in working memory $(n=4)$ and psychomotor function $(n=2)$, and only one patient had a deficit in verbal memory.

\section{Cognitive performance during $R T$ and concomitant TMZ}

During this phase of RT and concomitant TMZ treatment, 4 patients showed an improvement (previously defined as $z$ $\Delta \geq 1.5$ ) in one of the cognitive domains, 4 patients showed a deterioration (previously defined as $z \Delta \leq-1.5$ ) in one of the domains, 1 patient improved in one domain and deteriorated in another, and 4 patients had a stable performance on all six domains. None of the 13 patients showed a cognitive decline in more than one domain. At the end of this period (1st follow-up assessment), 9 patients had deficits in one $(n=3)$ or multiple $(n=6)$ cognitive domains.

\section{Cognitive performance during adjuvant TMZ}

During this phase of adjuvant TMZ treatment, 1 patient showed a deterioration in one of the cognitive domains, 1 patient showed a deterioration in two domains, and 11 patients remained completely stable on all six domains. None of the patients showed a deterioration in more than two cognitive domains. At the end of this period (2nd follow-up assessment), 11 patients showed deficits in one $(n=2)$ or multiple $(n=9)$ cognitive domains.

\section{Overall cognitive performance}

Overall, during 6 weeks of RT plus concomitant TMZ treatment and three cycles of adjuvant TMZ treatment, the performances of 7 patients remained stable in all six cognitive domains, the performances of 3 patients improved in one domain, the performances of 2 patients deteriorated in two domains, and the performances of 1 patient improved in one domain and deteriorated in another.

\section{Discussion}

This study is the first to prospectively examine cognitive functioning of GBM patients at different points in time during combined radio-chemotherapy treatment. Consistent with findings in the literature, the majority of the patients already had multiple cognitive deficits preceding RT treatment [3, 4]. The current findings suggest that, overall, cognitive functioning remains rather stable during treatment and that the addition of TMZ to RT does not necessarily lead to an additional deterioration in cognitive functioning during the first 6 months after diagnosis. For some patients, changes in cognitive functioning did occur. However, those patients had a deterioration in only one or two of the six cognitive domains assessed, and the number of patients showing cognitive decline was equal to the number of patients that cognitively improved. Less cognitive changes occur in the adjuvant TMZ treatment period compared to the RT and concomitant TMZ treatment period, suggesting that patients' cognitive functioning stabilizes in a less intense treatment period.

An earlier study, using the same neuropsychological test battery, reported that cognitive decline occurred in highgrade glioma patients during and following RT [7]. The present findings suggest that the addition of TMZ to RT, for GBM patients as a group, does not negatively affect cognitive functioning and might in fact even have a positive effect on cognitive performance, compared to the earlier mentioned cognitive decline of the patients that only received RT. These findings are also compatible with another recent study reporting that it is not the treatment, but the tumor itself and tumor recurrence, that are the largest determinants of cognitive decline [6].

To conclude, these initial results hold promise for the future use of combined treatment regimens as far as cognitive functioning is concerned. This study will continue with further patients, in order to draw more definite and detailed conclusions. Follow-up data on cognitive functioning will be collected after all six cycles of TMZ and 4 months after the sixth adjuvant TMZ cycle, to be able to determine possible late effects. 
Table $3 z$-scores and delta-scores

\begin{tabular}{|c|c|c|c|c|c|c|c|c|c|c|c|c|}
\hline \multirow[t]{2}{*}{ Case } & \multicolumn{4}{|c|}{ Information processing speed } & \multicolumn{4}{|c|}{ Psychomotor function } & \multicolumn{4}{|c|}{ Attention } \\
\hline & $\mathrm{B}(\mathrm{z})$ & $\Delta \mathrm{B}-\mathrm{FU} 1$ & $\Delta \mathrm{FU} 1-2$ & $\Delta \mathrm{B}-\mathrm{FU} 2$ & $\mathrm{~B}(\mathrm{z})$ & $\Delta \mathrm{B}-\mathrm{FU} 1$ & $\Delta \mathrm{FU} 1-2$ & $\Delta \mathrm{B}-\mathrm{FU} 2$ & $\mathrm{~B}(\mathrm{z})$ & $\Delta \mathrm{B}-\mathrm{FU} 1$ & $\Delta \mathrm{FU} 1-2$ & $\Delta \mathrm{B}-\mathrm{FU} 2$ \\
\hline 1 & -1.57 & -0.04 & 0.05 & 0.01 & -0.80 & 0.37 & 0.26 & 0.63 & -0.26 & 0.01 & -0.32 & -0.31 \\
\hline 2 & -2.01 & 0.49 & -0.39 & 0.10 & -0.68 & -1.51 & 0.73 & -0.78 & -2.71 & 1.19 & 0.10 & 1.29 \\
\hline 3 & & & & & & & & & -3.82 & -2.92 & & \\
\hline 4 & 0.45 & 0.41 & -0.71 & -0.30 & -1.06 & 1.28 & -1.33 & -0.05 & 0.67 & 0.45 & -0.24 & 0.21 \\
\hline 5 & -3.33 & 0.44 & -0.39 & 0.05 & 0.49 & -0.52 & 0.33 & -0.19 & -1.92 & 0.45 & -0.43 & 0.02 \\
\hline 6 & -3.69 & 0.60 & 0.39 & 0.99 & -1.93 & 1.22 & -1.33 & -0.11 & -4.44 & -0.10 & -0.33 & -0.43 \\
\hline 7 & $-\mathbf{3 . 1 0}$ & -0.01 & -0.82 & -0.83 & -1.00 & -1.80 & -1.70 & -3.50 & -0.86 & -0.86 & -1.64 & -2.50 \\
\hline 8 & -0.73 & 0.34 & -0.23 & 0.11 & 0.84 & -0.17 & 0.02 & -0.15 & -0.39 & -0.05 & 0.52 & 0.47 \\
\hline 9 & -0.13 & -0.50 & -0.23 & -0.73 & -1.35 & 0.26 & -0.47 & -0.21 & 0.07 & -0.27 & -0.48 & -0.75 \\
\hline 10 & -2.18 & -0.37 & -0.80 & -1.17 & -0.23 & 1.51 & 0.47 & 1.98 & -1.88 & -0.21 & -0.60 & -0.81 \\
\hline 11 & -1.01 & -1.13 & 0.40 & -0.73 & -0.09 & -1.04 & 0.33 & -0.71 & -1.52 & -2.16 & -0.40 & -2.56 \\
\hline 12 & -2.21 & 0.71 & -0.34 & 0.37 & 0.99 & -0.73 & -0.41 & -1.14 & -1.21 & 1.47 & -0.37 & 1.10 \\
\hline 13 & -1.50 & 0.07 & 0.05 & 0.12 & $-\mathbf{3 . 0 0}$ & 1.78 & -0.13 & 1.65 & -1.34 & 0.50 & -0.34 & 0.16 \\
\hline \multirow[t]{2}{*}{ Case } & \multicolumn{4}{|c|}{ Verbal memory } & \multicolumn{4}{|c|}{ Working memory } & \multicolumn{4}{|c|}{ Executive functioning } \\
\hline & $\mathrm{B}(\mathrm{z})$ & $\Delta \mathrm{B}-\mathrm{FU} 1$ & $\Delta \mathrm{FU} 1-2$ & $\Delta \mathrm{B}-\mathrm{FU} 2$ & B (z) & $\Delta \mathrm{B}-\mathrm{FU} 1$ & $\Delta \mathrm{FU1-2}$ & $\Delta \mathrm{B}-\mathrm{FU} 2$ & B (z) & $\Delta \mathrm{B}-\mathrm{FU} 1$ & $\Delta \mathrm{FU} 1-2$ & $\Delta \mathrm{B}-\mathrm{FU} 2$ \\
\hline 1 & 0.40 & 0.44 & -0.27 & 0.17 & -1.39 & 0.15 & 0.36 & 0.51 & -2.45 & 0.39 & -0.76 & -0.37 \\
\hline 2 & -0.05 & 0.02 & -0.17 & -0.15 & -1.11 & -0.22 & 0.49 & 0.27 & -1.42 & & 1.01 & -0.41 \\
\hline 3 & -0.76 & -0.65 & 0.52 & -0.13 & -1.32 & 0.16 & -0.70 & -0.54 & -5.24 & & & \\
\hline 4 & -0.80 & 0.19 & 0.83 & 1.02 & 0.53 & 0.43 & -0.49 & -0.06 & -1.31 & 2.24 & -0.54 & 1.70 \\
\hline 5 & -0.85 & 0.66 & 0.38 & 1.04 & -1.75 & 0.32 & -1.11 & -0.79 & -2.72 & 1.52 & -0.79 & 0.73 \\
\hline 6 & -0.57 & -0.02 & -0.21 & -0.23 & -2.63 & 1.40 & -0.73 & 0.67 & -4.40 & 1.36 & 0.78 & 2.14 \\
\hline 7 & -1.49 & 0.09 & 0.41 & 0.50 & -2.88 & -1.03 & -0.38 & -1.41 & -2.24 & -0.37 & -0.40 & -0.77 \\
\hline 8 & 0.07 & 0.24 & 0.43 & 0.67 & -0.87 & -0.17 & -0.05 & -0.22 & -0.23 & -0.58 & 0.97 & 0.39 \\
\hline 9 & 0.35 & -0.25 & 0.19 & -0.06 & 0.10 & -0.20 & -0.07 & -0.27 & -2.00 & 0.61 & 0.07 & 0.68 \\
\hline 10 & -3.28 & 0.20 & 0.91 & 1.11 & 1.02 & -0.43 & -0.26 & -0.69 & -3.43 & -2.30 & 0.39 & -1.91 \\
\hline 11 & 0.35 & -0.19 & -0.11 & -0.30 & -0.19 & -0.77 & 0.57 & -0.20 & -1.13 & -0.33 & -1.41 & -1.74 \\
\hline 12 & -0.99 & 0.16 & -0.10 & 0.06 & -1.96 & 1.65 & -1.63 & 0.02 & -2.08 & 1.25 & -0.80 & 0.45 \\
\hline 13 & 0.05 & 0.41 & 0.05 & 0.46 & -0.91 & 0.27 & 0.42 & 0.69 & -1.25 & 0.19 & -0.66 & -0.47 \\
\hline
\end{tabular}

Individual $z$-scores and delta $(\Delta)$-scores per patient for all six cognitive domains. Performance is relative to that of age, gender, and educationmatched healthy controls. A higher baseline $z$-score means better performance. A negative delta score indicates deterioration, a positive delta score indicates amelioration. Significant deviations [individual $z$-scores of 1.5 or more below that of healthy controls $(z \leq-1.5)$ ] and changes [individual changes of $1.5 z$-score or more $(z \Delta \geq 1.5$ or $z \Delta \leq-1.5)$ ] are depicted in bold.

$B$ Baseline (after surgery, but before RT and concomitant TMZ), FU1 follow-up 1 (after 6 weeks of conventional RT and concomitant TMZ, but before adjuvant TMZ), FU2 follow-up 2 (after three cycles of adjuvant TMZ)

For now, the finding that cognitive functioning does not deteriorate during RT and TMZ combined treatment is of importance for clinical practice. For those professional caregivers involved in the treatment of GBM patients, it is important to recognize that combined modality treatment not only seems to be safe in terms of health-related quality of life, but also in terms of cognitive functioning.

Acknowledgments We thank Lies Braam for her participation in tracing patients and Wilmy Cleijne for her invaluable help in testing patients.
Open Access This article is distributed under the terms of the Creative Commons Attribution Noncommercial License which permits any noncommercial use, distribution, and reproduction in any medium, provided the original author(s) and source are credited.

\section{References}

1. Stupp R, Mason WP, van den Bent MJ et al (2005) Radiotherapy plus concomitant and adjuvant temozolomide for glioblastoma. $\mathrm{N}$ Engl J Med 352:987-996 
2. Taphoorn MJ, Klein M (2004) Cognitive deficits in adult patients with brain tumours. Lancet Neurol 3:159-168

3. Tucha O, Smely C, Preier M, Lange KW (2000) Cognitive deficits before treatment among patients with brain tumors. Neurosurgery 47:324-333

4. Klein M, Taphoorn MJ, Heimans JJ et al (2001) Neurobehavioral status and health-related quality of life in newly diagnosed highgrade glioma patients. J Clin Oncol 19:4037-4047

5. Schmidinger M, Linzmayer L, Becherer et al (2003) Psychometric- and quality-of-life assessment in long-term glioblastoma survivors. J Neurooncol 63:55-61

6. Brown PD, Jensen AW, Felten SJ et al (2006) Detrimental effects of tumor progression on cognitive function of patients with highgrade glioma. J Clin Oncol 24:5427-5433

7. Bosma I, Vos MJ, Heimans JJ et al (2007) The course of neurocognitive functioning in high-grade glioma patients. Neuro-oncology 9:53-62
8. Correa DD, Maron L, Harder $\mathrm{H}$ et al (2007) Cognitive functions in primary central nervous system lymphoma: literature review and assessment guidelines. Ann Oncol 18(7):1145-1151

9. Taphoorn MJ, Stupp R, Coens C et al (2005) Health-related quality of life in patients with glioblastoma: a randomised controlled trial. Lancet Oncol 6:937-944

10. Lezak MD (1995) Neuropsychological assessment. Oxford University Press, New York

11. Houx PJ, Jolles J (1994) Vulnerability factors for age-related cognitive decline. In: Isaacson RL, Jensen KF (eds) The vulnerable brain and environmental risks. Plenum, New York, pp 25-41

12. Sternberg S (1975) Memory scanning: new findings and current controversies. Q J Exp Psychol 27:1-32 